\title{
Formation and decay of super heavy systems
}

\author{
Toshiki Maruyama ${ }^{\mathrm{a}, \mathrm{b}}$ Aldo Bonasera ${ }^{\mathrm{a}}$ Massimo Papa ${ }^{\mathrm{a}, \mathrm{c}}$ \\ Satoshi Chiba ${ }^{\mathrm{b}}$ \\ a Istituto Nazionale Fisica Nucleare-Laboratorio Nazionale del Sud, Via Santa \\ Sofia 44, Catania 95123, Italy \\ b Advanced Science Research Center, Japan Atomic Energy Research Institute, \\ Tokai, Ibaraki 319-1195, Japan \\ ${ }^{c}$ Istituto Nazionale Fisica Nucleare-Sezione di Catania, Corso Italia 57, Catania \\ 95129, Italy
}

\begin{abstract}
We investigate the formation and the decay of heavy systems which are above the fission barrier. By using a microscopic simulation of constraint molecular dynamics (CoMD) on $\mathrm{Au}+\mathrm{Au}$ collision, we observe composite states stay for very long time before decaying by fission.
\end{abstract}

The typical reaction mechanisms of heavy-ion collisions at lower incident energy are, depending on the energy and impact parameters, complete fusion, incomplete fusion, fusion-fission, molecular resonance, and deep inelastic collisions. Among the huge amount of studies in this field, collisions of very heavy nuclei have been investigated mainly for the creation of super heavy element (SHE). SHEs are produced in two ways: one is "cold fusion" which is complete fusion below the classical barrier, and the other is "hot fusion" which allows several neutrons to be emitted. Even though the name is "hot", such reactions are still at very low energy near the barrier and the total mass number is very close to the aimed one. As far as the formation of SHE is concerned, the "fusion" of very heavy nuclei where the fission barrier no more exists is found to be ineffective $[1,2]$.

Apart from the formation of SHE, the study of fission dynamics, including the spontaneous fission and the fusion-fission of heavy composite, has been one of the most important subjects. The competition of neutron emission between the

Email addresses: maru@hadron02.tokai.jaeri.go.jp (Toshiki Maruyama), bonasera@lns.infn.it (Aldo Bonasera), papa@lns.infn.it (Massimo Papa), chiba@hadron31.tokai.jaeri.go.jp (Satoshi Chiba).

Preprint submitted to Elsevier Science 24 October 2018 
fission and the fission delay have been discussed intensively. However almost all the discussion are done for mass regions where the classical fission barrier exists.

Sometime ago many physicists paid attention to the low energy collision of very heavy nuclei in regard to the spontaneous positron emission from strong electric fields [3]. If a molecule state of, say, $U$ and $U$ is formed and stays sufficiently long time, the binding energy of a electron can exceed the electron mass and might create electron-positron pair by a static QED process. Unfortunately no clear evidence of static positron creation was observed below Coulomb energy region. They have pointed out [4] the importance of nuclear reaction which causes the time delay of separation of two nuclei. Although there increases the background component of positrons from nuclear excitation, which in this case is not interested in, the electron-positron from the static QED process is also expected to increase. However, the reaction mechanism of very heavy nuclei has not been discussed by fully dynamical models.

In this paper we discuss the possibility of molecule-like states of heavy nuclei and the time scale of very heavy composite system formed by the fusionfission or deep inelastic processes. To investigate these problems theoretically we use a recently developed constraint molecular dynamics (CoMD) model [5]. This model has been proposed to include the Fermionic nature of constituent nucleons by a constraint that the phase space distribution should always satisfy the condition $f \leq 1$. Among similar molecular dynamics models, there are quantum molecular dynamics (QMD) [6], Fermionic molecular dynamics (FMD) [7], and antisymmetrized molecular dynamics (AMD) [8]. QMD has been the most popular and feasible model. Unfortunately it cannot, in principle, deal with the Fermionic nature of nuclear system, although sometime the phenomenological Pauli potential is introduced for such a purpose. Therefore QMD model has been used mainly for higher energy phenomena except for some exceptions $[9,10]$. More sophisticated models, i.e. FMD and AMD, deal with antisymmetrization of the wave function and have succeeded in describing nuclear reactions at medium low energy and also in the study of nuclear structures. However, due to the four dimensional matrix element of two-body interaction, the CPU time necessary to work out calculations for systems with total mass larger than 200 is very large for practical studies. The constraint molecular dynamics, on the other hand, can deal to a certain extent with the Fermionic nature of the nuclear systems and it is still feasible for heavy systems.

In this paper we apply CoMD to the investigation of ${ }^{197} \mathrm{Au}+{ }^{197} \mathrm{Au}$ collisions at low energies where fusion-fission or deep-inelastic process may occur. In the following we give a brief review of the model [5].

The CoMD model mainly consists of two parts: classical equation of motion of 
many-body system, and stochastic process which includes constraint of Pauli principle and the two-body collisions. We write the distribution function of the system as a sum of one-body distribution function neglecting the antisymmetrization

$$
\begin{aligned}
f(\mathbf{r}, \mathbf{p}) & =\sum_{i} f_{i}(\mathbf{r}, \mathbf{p}) \\
f_{i}(\mathbf{r}, \mathbf{p}) & =\frac{1}{\left(2 \pi \sigma_{r} \sigma_{p}\right)^{3}} \cdot \exp \left[-\frac{\left(\mathbf{r}-\left\langle\mathbf{r}_{i}\right\rangle\right)^{2}}{2 \sigma_{r}{ }^{2}}-\frac{\left(\mathbf{p}-\left\langle\mathbf{p}_{i}\right\rangle\right)^{2}}{2 \sigma_{p}{ }^{2}}\right]
\end{aligned}
$$

The equation of motion of $\left\langle\mathbf{r}_{i}\right\rangle$ and $\left\langle\mathbf{p}_{i}\right\rangle$ are derived using the time-dependent variational principle which gives:

$$
\left\langle\dot{\mathbf{r}_{i}}\right\rangle=\frac{\partial H}{\partial\left\langle\mathbf{p}_{i}\right\rangle}, \quad\left\langle\dot{\mathbf{p}}_{i}\right\rangle=-\frac{\partial H}{\partial\left\langle\mathbf{r}_{i}\right\rangle} .
$$

In our approach the total energy $H$ for $A$ particles with mass $m$ consists of the kinetic energy and the effective interactions:

$$
H=\sum_{i} \frac{\left\langle\mathbf{p}_{i}\right\rangle^{2}}{2 m}+A \frac{3 \sigma_{p}^{2}}{2 m}+V
$$

The second term arises from the Gaussian width in p-space. However in the following considerations we omit such a constant term.

The effective interaction $V$ we adopt is written as

$$
V=V^{\mathrm{vol}}+V^{(3)}+V^{\mathrm{sym}}+V^{\mathrm{surf}}+V^{\mathrm{Coul}} .
$$

By defining the superimposition integral $\rho_{i j}$ as:

$$
\begin{aligned}
\rho_{i j} & \equiv \int d^{3} r_{i} d^{3} r_{j} \rho_{i}\left(\mathbf{r}_{i}\right) \rho_{j}\left(\mathbf{r}_{j}\right) \delta\left(\mathbf{r}_{i}-\mathbf{r}_{j}\right) \\
\rho_{i} & \equiv \int d^{3} p f_{i}(\mathbf{r}, \mathbf{p})
\end{aligned}
$$

the terms in Eq. (5) can be written as:

$$
\begin{aligned}
V^{\mathrm{vol}} & =\frac{t_{0}}{2 \rho_{0}} \sum_{i, j \neq i} \rho_{i j}, \\
V^{(3)} & =\frac{t_{3}}{(\mu+1)\left(\rho_{0}\right)^{\mu}} \sum_{i, j \neq i} \rho_{i j}^{\mu},
\end{aligned}
$$




$$
\begin{aligned}
V^{\mathrm{sym}} & =\frac{a_{\mathrm{sym}}}{2 \rho_{0}} \sum_{i, j \neq i}\left[2 \delta_{\tau_{i}, \tau_{j}}-1\right] \rho_{i j}, \\
V^{\mathrm{surf}} & =\frac{C_{s}}{2 \rho_{0}} \sum_{i, j \neq i} \nabla_{\left\langle\mathbf{r}_{i}\right\rangle}^{2}\left(\rho_{i j}\right), \\
V^{\text {Coul }} & =\frac{1}{2} \sum_{\substack{i, j \neq i \\
(i, j \in \text { protons })}} \frac{e^{2}}{\left|\left\langle\mathbf{r}_{i}\right\rangle-\left\langle\mathbf{r}_{j}\right\rangle\right|} \operatorname{erf}\left(\frac{\left|\left\langle\mathbf{r}_{i}\right\rangle-\left\langle\mathbf{r}_{j}\right\rangle\right|}{2 \sigma_{r}^{2}}\right) .
\end{aligned}
$$

In the above relations the coordinate $\tau_{i}$ represents the nucleon isospin degree of freedom.

We have two sets of parameters, regarding the interaction strength and the width of distribution function, which are different mainly for the stability of the system.

The parameter set (I) used in [5], $\sigma_{r}=1.3 \mathrm{fm}, \sigma_{p} / \hbar=0.47 \mathrm{fm}^{-1}, t_{0}=-356$ $\mathrm{MeV}, t_{3}=303 \mathrm{MeV}, \mu=7 / 6, a_{\mathrm{sym}}=32 \mathrm{MeV}, C_{s}=-0.33 \mathrm{MeV} \cdot \mathrm{fm}^{2}, \rho_{0}=$ $0.165 \mathrm{fm}^{-3}$, gives a good reproduction of fragmentation data on $\mathrm{Ca}+\mathrm{Ca}$ and $\mathrm{Au}+\mathrm{Au}$ at $35 \mathrm{MeV} /$ nucleon, and the mean radii and the binding energies in a wide range of mass.

The parameter set (II), which we introduce in this paper, $\left(\sigma_{r}=1.15 \mathrm{fm}\right.$, $\sigma_{p} / \hbar=0.4748 \mathrm{fm}^{-1}, t_{0}=-301.1 \mathrm{MeV}, t_{3}=242 \mathrm{MeV}, \mu=7 / 6, a_{\text {sym }}=26.4$ $\mathrm{MeV}, C_{s}=-0.165 \mathrm{MeV} \cdot \mathrm{fm}^{2}$, and $\rho_{0}=0.165 \mathrm{fm}^{-3}$ ), reproduces reasonably well the fusion cross section of $\mathrm{Ca}+\mathrm{Ca}$ reactions, while set (I) overestimates such a data. Even though we have not been able yet to find a unique parameter set consistent with both features of fusion and fragmentation, we find some experimental confirmations of our calculations as mentioned above. In this work, since we apply the model to an energy region and to very heavy systems for which the experimental data is scarce, we plan to give upper and lower estimates which will be interesting to confirm experimentally. We further strengthen our results with Boltzmann-Nordheim-Vlasov (BNV) calculations[11].

The Pauli principle is taken into account in two ways: One is the Pauli blocking of the final state of two-body collision and the other is the constraint which brings into the system the Fermi motion in a stochastic way. The starting point of the constraint is the requirement:

$$
\begin{aligned}
& \bar{f}_{i} \leq 1 \quad(\text { for all } i), \\
& \bar{f}_{i} \equiv \sum_{j} \delta_{\tau_{i}, \tau_{j}} \delta_{s_{i}, s_{j}} \int_{h^{3}} f_{j}(\mathbf{r}, \mathbf{p}) d^{3} r d^{3} p,
\end{aligned}
$$

where $s_{i}$ is the spin coordinate of the nucleon $i$. The integral is performed in an hypercube of volume $h^{3}$ in the phase-space centered around the point 
$\left(\left\langle\mathbf{r}_{i}\right\rangle,\left\langle\mathbf{p}_{i}\right\rangle\right)$ with size $\sqrt{\frac{2 \pi \hbar}{\sigma_{r} \sigma_{p}}} \sigma_{r}$ and $\sqrt{\frac{2 \pi \hbar}{\sigma_{r} \sigma_{p}}} \sigma_{p}$ in the $r$ and $p$ spaces respectively.

At each time step and for each particle $i$ the phase space occupation $\bar{f}_{i}$ is checked. If $\bar{f}_{i}$ has a value greater than 1 an ensemble $K_{i}$ of nearest particles (including the particle $i$ ) is determined within the distances $3 \sigma_{r}$ and $3 \sigma_{p}$ in the phase space. Then we change randomly the momenta of the particles belonging to the ensemble $K_{i}$ in such a way that for the newly generated sample the total momentum and the total kinetic energy is conserved ("manybody elastic scattering"). The new sample is accepted only if it reduces the phase space occupation $\bar{f}_{i}[5]$.

To handle the Pauli-blocking in the collision term is straightforward from the constraint. In fact for each NN collision we evaluate the occupation probability after the elastic scattering. If such functions are both less than 1 the collision is accepted, rejected otherwise. We note that for the results discussed here and especially at the lowest energies the collision term is of little importance.

To simulate the collision of two ${ }^{197} \mathrm{Au}$ nuclei, we prepare the ground state by applying the frictional cooling method together with the constraint of CoMD. The ground states we obtain have binding energy of $7.6 \mathrm{MeV} /$ nucleon and a root mean square radius of $5.76 \mathrm{fm}$ with parameter set (I) and 8.4 $\mathrm{MeV} /$ nucleon and $5.34 \mathrm{fm}$ for parameter set (II). They are rather stable for $1000 \mathrm{fm} / c$. For instance our ${ }^{197} \mathrm{Au}$ ground states with parameter sets (I) and (II) evaporates 2.75 and 3.1 nucleons during $1000 \mathrm{fm} / c$, respectively. The collision events are performed for impact parameter $b$ of 0 and $6 \mathrm{fm}$ for incident energy in laboratory system of $E_{\text {lab }}=5 \sim 35 \mathrm{MeV} /$ nucleon.

Figure 1 shows a typical event of CoMD (I) calculation with incident energy $E_{\text {lab }}=10 \mathrm{MeV} /$ nucleon with impact parameter $b=6 \mathrm{fm}$. The two nuclei form a quite deformed compound system, they keep such a deformation almost $2500 \mathrm{fm} / c$ and finally fission takes place. The system does not show much rotation since the angular momentum per nucleon is not so large and the elongated shape makes the moment of inertia larger than that in the initial stage. Therefore the reaction mechanism we are observing here may be inbetween the deep inelastic and molecular resonance.

There are many observables which distinguish the reaction mechanism. The largest fragment mass is one of such well-defined observables which can easily be measured experimentally. Figure 2 shows the time dependences of the largest cluster mass for the impact parameters $b=0$ and $6 \mathrm{fm}$ calculated by CoMD (I), CoMD (II), QMD and BNV. In CoMD calculations we see at the beginning the largest cluster mass $A_{\max }=197$ which corresponds to projectile and target mass number. Within about $50 \mathrm{fm} / c, A_{\max }$ becomes 394 except for the incident energy $E_{\text {lab }}=5 \mathrm{MeV}$ which is below the barrier where two nuclei never contact. At incident energies above the barrier, the formed 

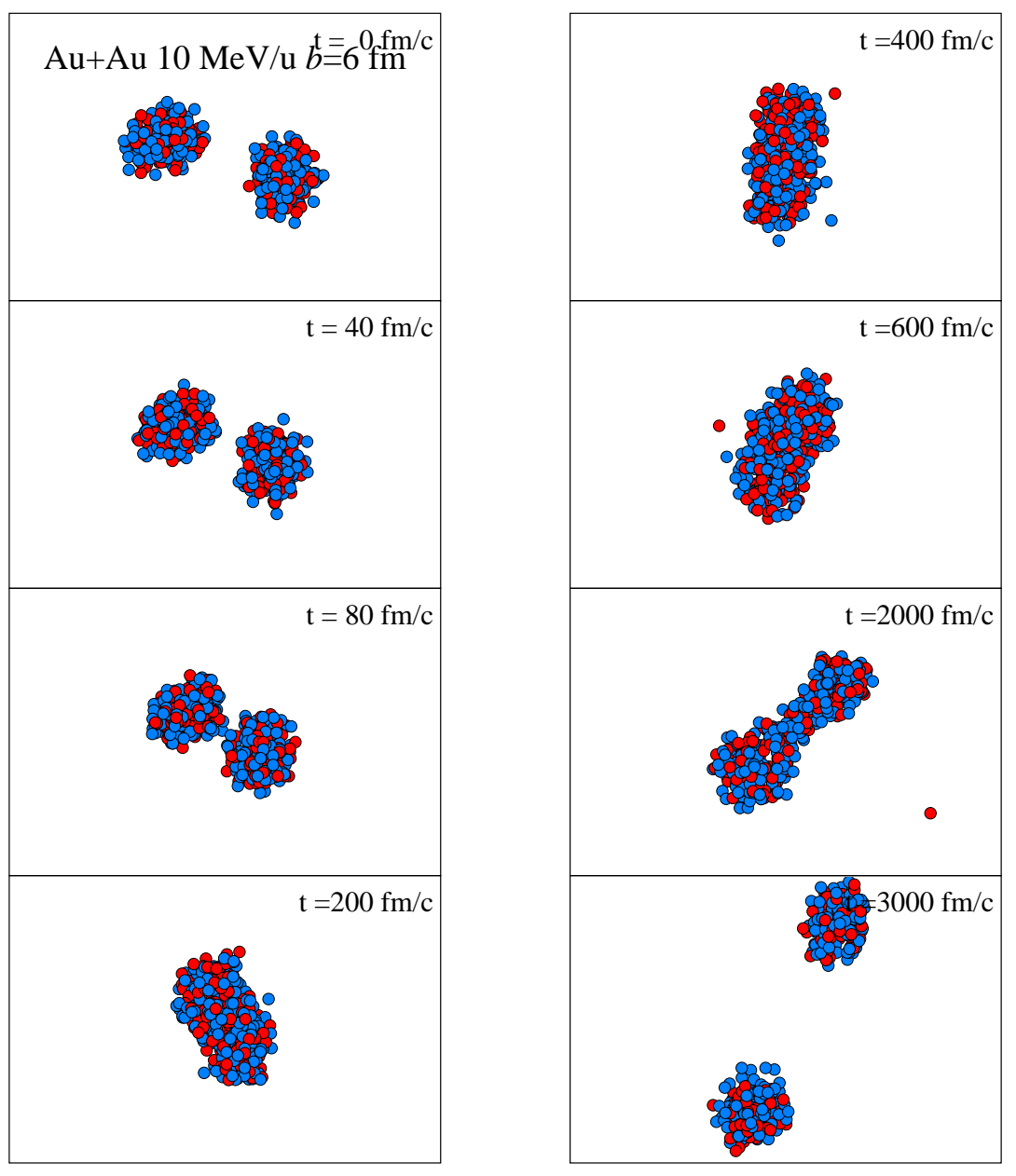

Fig. 1. Snapshot of ${ }^{197} \mathrm{Au}+{ }^{197} \mathrm{Au}$ at $E_{\text {lab }}=10 \mathrm{MeV} /$ nucleon $b=6 \mathrm{fm}$. The time indicated in each panel is not from the contact of two nuclei but indicates only that of the simulation.

large system will decay into smaller fragments by different modes according to the energy and angular momentum. At higher incident energies $\left(E_{\text {lab }} \geq 30\right.$ $\mathrm{MeV} /$ nucleon) the largest cluster mass changes suddenly at the early stage and continuously decreases in time. This indicates multifragmentation for head-on collisions and deep inelastic reaction for peripheral collisions followed by the emission of nucleons and small fragments. At lower incident energies $\left(E_{\text {lab }} \leq 20\right.$ $\mathrm{MeV} /$ nucleon) there is a sudden change of the largest cluster mass at very late time, which indicates a fission of the system. One should note that in our calculation of $\mathrm{Au}+\mathrm{Au}$ system there is almost no event where the system decays only by emitting particles or light fragments, i.e., pure incomplete fusion. The instability due to the Coulomb repulsion plays the major role in the decay process. 


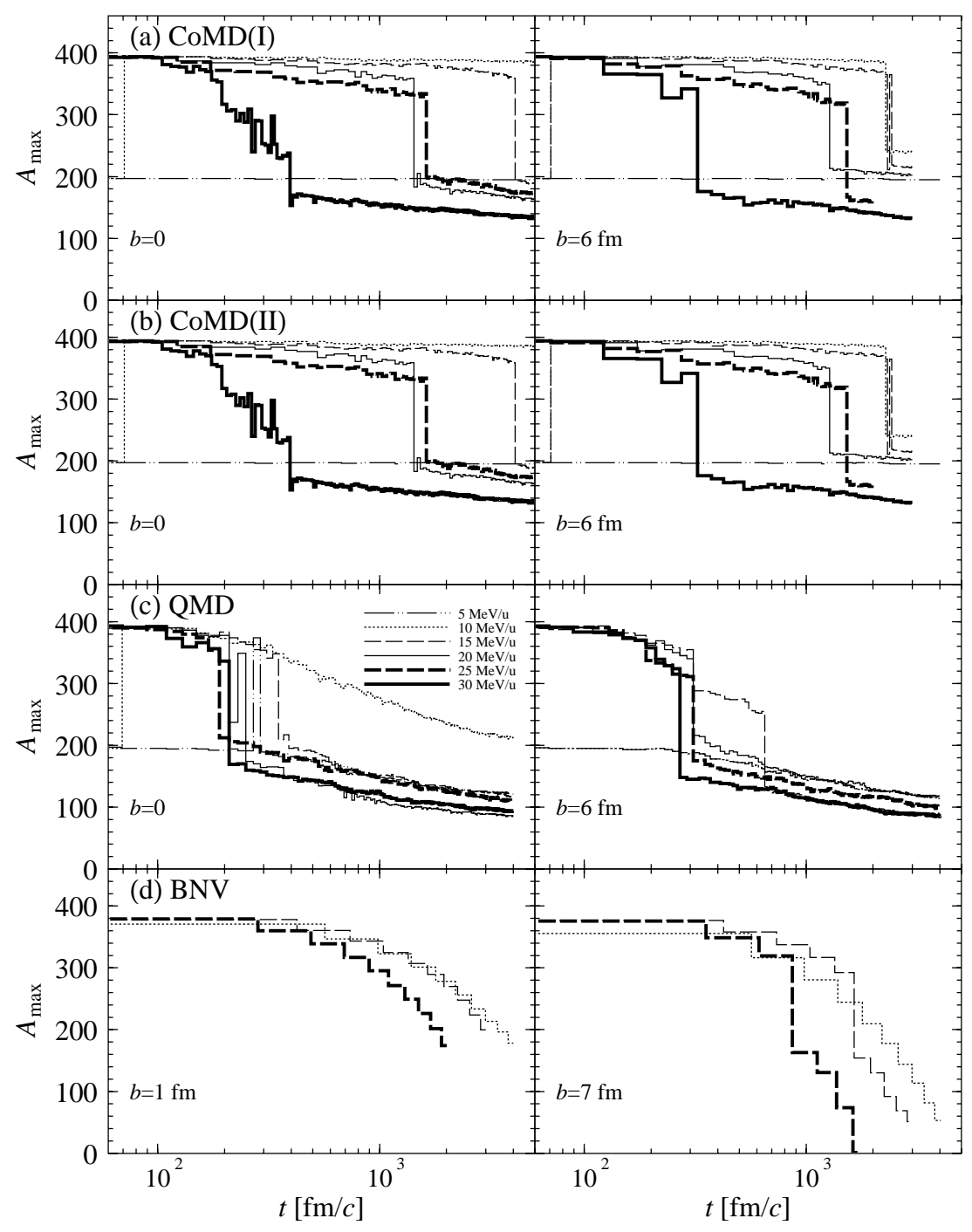

Fig. 2. The time-dependence of the largest fragment mass $A_{\max }$. From the top (a) CoMD with parameter set (I), (b) CoMD (II), and (c) QMD. The left panels show cases of head-on collision and the right $b=6 \mathrm{fm}$. The lowest panels (d) refer to BNV calculations at 1 and $7 \mathrm{fm}$ respectively.

Here we should note that the plotted largest fragment mass are obtained by only one event for each incident energy and impact parameter. Therefore the fission time includes large amount of statistical error. In fact the case of $E_{\text {lab }}=10 \mathrm{MeV} /$ nucleon with $b=0 \mathrm{fm}$, fission process is not observed in Fig. 2 (a). With a different series of initial conditions, however, we observe fission of the system around $t=10^{4} \mathrm{fm} / c$ for parameter (I). In Fig. 2 (c) the same quantity as Fig. 2 (a) and (b) is displayed for QMD calculation. These QMD calculations are based on the same code as CoMD (I) switching off the constraint procedure. The difference between the CoMD and the QMD 
is clear and dramatic. At low energy collisions there are no fission process and the system decays only by emitting nucleons and light fragments. At higher energies there is some sudden change of the largest fragment mass even in QMD calculation. This is not a fission but passing through for head-on collisions or deep inelastic process for peripheral collisions.

In BNV [11] calculation (Fig. 2 (d)) the sudden change of maximum fragment mass number is not observed for impact parameter $b=1 \mathrm{fm}$ and $b=7 \mathrm{fm}$ at low energy, except for $b=7 \mathrm{fm}$ and $E_{\text {lab }} \geq 15 \mathrm{MeV} /$ nucleon. For the fission process with very small angular momentum, fluctuations and correlations are very important which are not included in the BNV calculation. Instead, the system decays via evaporation of nucleons like the QMD case. One should note, however, that the Pauli principle is satisfied in BNV calculation while it is not in QMD case. The time scale of the very large composite is still of the same order as in CoMD (II) calculation. Although the reaction mechanism is different for CoMD and BNV, this similarity of time scale supports the validity of our CoMD calculation.

Assuming a very simple form of the time-dependent fission width $\Gamma(t)=$ $\Gamma_{\mathrm{f}} \theta\left(t-T_{\mathrm{d}}\right)$, the averaged fission time $T_{\text {fiss }}$ can be obtained by the survival probability of the compound system against two-body process $P_{\text {surv }}$ as

$$
\begin{aligned}
P_{\text {surv }} & =\exp \left[-\left(t-T_{\mathrm{d}}\right) \Gamma_{\mathrm{f}} / \hbar\right] \\
T_{\text {fiss }} & \equiv T_{\mathrm{d}}+\hbar / \Gamma_{\mathrm{f}}
\end{aligned}
$$

where $T_{\mathrm{d}}$ is the delay time and $\Gamma_{\mathrm{f}}$ is the "fission width" after the delay time. The probability $P_{\text {surv }}(t)$ is obtained directly by the simulation. This fitting can apply well only for fission-like process in our calculation. Figure 3 shows the survival probability of a large fragment with $A>350$ which decays by fission-like mode or emitting large fragments $(A>30)$. The histograms are directly obtained by the simulation and the curves are fitting by Eqs. (1516). From the top, results of CoMD with parameter set (I), CoMD (II) and QMD are listed for impact parameter $b=0$ and for several incident energies $E_{\text {lab }}=10 \sim 25 \mathrm{MeV} /$ nucleon. For all the calculations the fitting works well, particularly the effect of delay time. The assumption of constant fission width after the delay time, on the other hand, is not completely supported because of poor statistics and still existing dynamical effects. One should note that the fitting by Eqs. (15-16) is just to extract the "fission" time of the super heavy composite. Especially the time scale of QMD results is obviously different from that of fission process.

The extracted fission time $T_{\text {fiss }}$ are plotted in Fig. 4. The fission time shown for parameter set (I) might be too long for such heavy system. To make more quantitative discussions we should improve the effective interaction. By using parameter set (II), we obtain smaller values of fission time. We can consider 


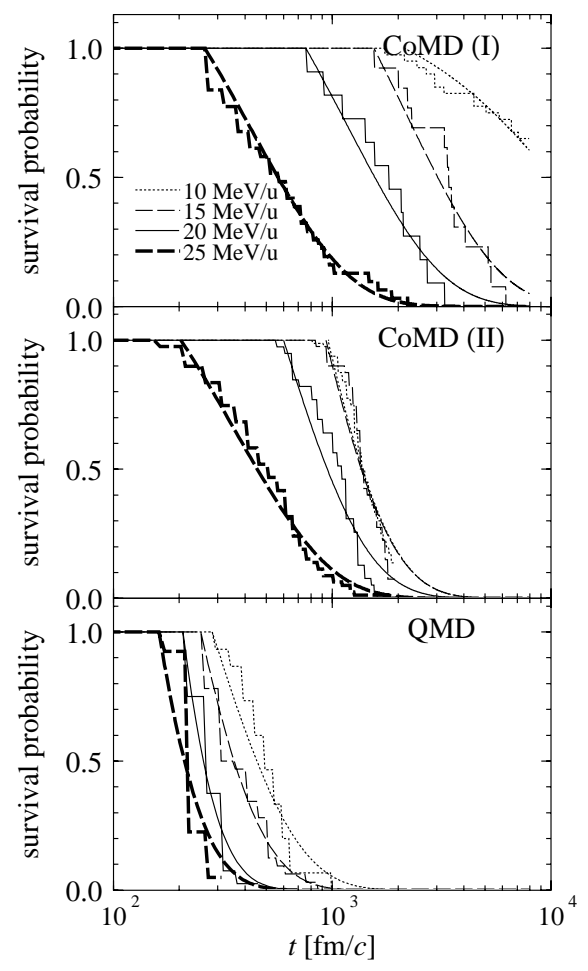

Fig. 3. The survival probability of large fragments $(A>350)$ which decay by fission-like mode. The abscissa indicates the time after the contact of two nuclei. From the top the results are obtained for head-on collisions by CoMD with parameter set (I), CoMD (II), and QMD. The histograms indicate results from simulations and smooth curves are the fits by Eqs. (15-16).

the values obtained as upper- and lower-limits of the fission time in our CoMD model. However the experimental data will finally support one or the other result which, we stress, are both qualitatively similar and somehow surprising. For both of parameter sets the longest life time of very heavy composite is found at $E_{\text {lab }}=10 \mathrm{MeV} /$ nucleon.

For lower incident energies (just above the Coulomb barrier) the system might not form a fully thermalized single composite but might be quasi separated in the phase space, which makes the system split easily. For higher energies, the fully thermalized system needs some fluctuations to reseparate even though there is no classical barrier for fission. Therefore the fission time gets shorter with increase of the incident energy.

In QMD calculation what is in marked contrast to the CoMD calculation is that the "fission time" has no maximum energy and shows monotonic decrease. This is due to the lack of the Pauli principle which suppresses two nuclei from overlapping at very low energies (above the Coulomb barrier). 


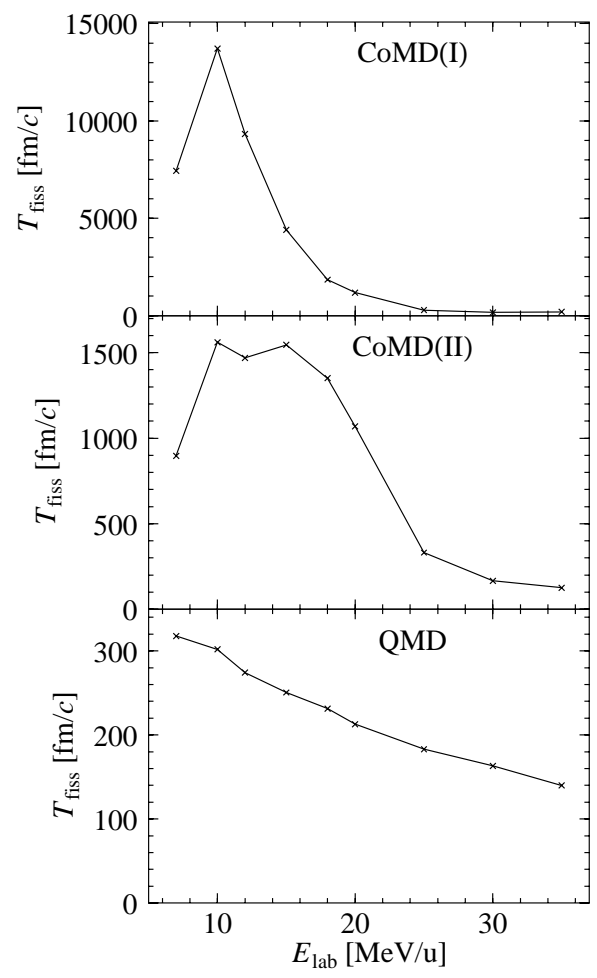

Fig. 4. The fission life time obtained from the Eqs. (15-16).

For peripheral collision ( $b=6 \mathrm{fm}$ ), the life time of very heavy composite is shorter than the head-on collisions. But the incident-energy dependence is very similar to the $b=0 \mathrm{fm}$ cases. Though the mechanism is much more dynamical, Eqs. (15-16) fit well again.

Nevertheless, the super heavy composite system formed by the head-on collision of $A u+A u$ may survive rather long time of $10^{3} \sim 10^{4} \mathrm{fm} / \mathrm{c}$. We note that such long-lived strongly deformed (see Fig. 1) systems have been observed by looking at the binary dissipative collisions between lighter system $\left(A_{\text {total }} \simeq\right.$ $60)$ in the same scaled energy regime with respect to the Coulomb barrier. This long time intervals have been well estimated through the comparison of the incident-energy averaged angular distributions and/or the excitation functions with the results of the partially overlapped molecular level model (POMLM)[12]. Such studies could be surely extended, at least concerning the average angular distribution of the binary processes, also in the present case.

Moreover, another interesting aspect of the long-lived very heavy system is, as mentioned before, the spontaneous positron-electron production from the strong electric field as a static QED process. The total charge of $\mathrm{Au}+\mathrm{Au}$ system may be still smaller than the necessary charge $(Z \sim 170)$ for this process. However, the nuclear reaction of, e.g. U+U system, should be qualitatively 


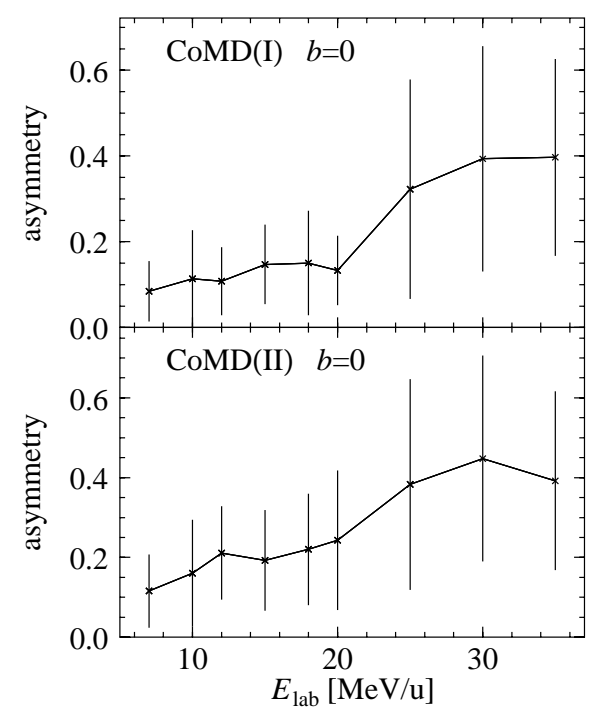

Fig. 5. The mass asymmetry of the fission fragments. Error bars indicate statistical standard deviation.

the same as what we observe in $\mathrm{Au}+\mathrm{Au}$ system. Although the background positrons should be larger, one can get longest life time of strong electric field (stronger than the case of Rutherford or molecular trajectory) around $E_{\text {lab }}=10 \mathrm{MeV} /$ nucleon at some impact parameter and the production of positrons from the static QED process should be largest around that energy.

As mentioned above, production of SHE is one of the most important subject in the heavy-ion collision problem. Besides cold- and hot-fusion, mass transfer in collision of very heavy nuclei was tried before. One could produce, e.g. up to $\operatorname{Fm}(Z=100)$ in $\mathrm{U}+\mathrm{U}$ system, or $\mathrm{Md}(Z=101)$ in $\mathrm{U}+\mathrm{Cm}$ system, by such a mechanism $[1,2]$. The incident energy, however, was very close to the Coulomb barrier and the reaction was rather gentle with the transfer of $\sim 20$ nucleons. In our CoMD calculation for $E_{\text {lab }} \geq 7 \mathrm{MeV} /$ nucleon, the reaction mechanism is more violent and there happens the transfer of much more nucleons though the mass loss from the system is also large. In Fig. 5 plotted is the massasymmetry $\left(A_{1}-A_{2}\right) /\left(A_{1}+A_{2}\right)$ of the fission process in CoMD calculation, where $A_{1}$ and $A_{2}$ are the largest and the second largest fragment mass when the fission occurs. The mass-asymmetry increases with the incident energy. At $E_{\text {lab }}=7 \mathrm{MeV} /$ nucleon, the asymmetry amounts to about 0.1 and at 10 $\mathrm{MeV} /$ nucleon almost 0.2 as average. If we simply assume no proton loss and asymmetry of 0.2 the largest fragment charge will be 112 for $\mathrm{U}+\mathrm{U}$ system. Of course we should consider the thermal mass loss and subsequent fission due to the excitation of fragments. However, such kind of fusion-fission mechanism at around $10 \mathrm{MeV} /$ nucleon should be taken into account for the SHE production. The new $4 \pi$ detectors can accumulate lots of statistics plus they can make coincidence studies to see if the fragments come from fission. 
In summary, we have discussed the formation and decay of super heavy composite in the $\mathrm{Au}+\mathrm{Au}$ collisions. The $\mathrm{CoMD}$ calculation which takes into account the Fermionic nature of the nucleon many-body system can describe well the low-energy dynamics including fusion, fission, deep inelastic, emission of nucleons and small fragments, and multifragmentation. Although there are still some ambiguities on the effective interaction, the life time of super heavy composite is found to be rather long up to $10^{3} \sim 10^{4} \mathrm{fm} / \mathrm{c}$. Some experimental explorations such as detection of $e^{+} e^{-}$formation at around $10 \mathrm{MeV} /$ nucleon and measurement of the energy averaged angular distribution and/or excitation function for binary processes are encouraged.

One of the authors T.M. thanks INFN-LNS for warm hospitality during his stay and Dr. A. Iwamoto, Dr. H. Ikezoe, and Dr. S. Mitsuoka for fruitful discussions. A.B. thanks Prof. J. Natowitz for enlighting discussion on the super heavy system discussed here.

\section{References}

[1] H. Gäggeler et al., Proc. 4th Int. Conf. on Nuclei Far From Stability, L.O. Skolen, Helsinger 1981, CERN 81-09, p 763.

[2] G. Herrmann, Proc. 4th Int. Conf. on Nuclei Far From Stability, L.O. Skolen, Helsinger 1981, CERN 81-09, p 772.

[3] "Quantum Electrodynamics of Strong Fields", ed. W. Greiner, Plenum Press New York and London, 1983; R. Bär et al, Nucl. Phys. A583 (1995) 237; S.M Ahmad et al, Nucl. Phys. A583 (1995) 247; A. Lépine-Szily et al, Nucl. Phys. A583 (1995) 263.

[4] J. Reinhardt, in "Quantum Electrodynamics of Strong Fields", ed. W. Greiner, Plenum Press New York and London, 1983.

[5] M. Papa, T. Maruyama and A. Bonasera, Phys. Rev. C. August 2001, in press.

[6] For review, see J. Aichelin, Phys. Rep. 202, (1991) 233.

[7] H. Feldmeier and Jurgen Schnack, Rev. Mod. Phys 72, (2000) 655.

[8] A. Ono, H. Horiuchi, T. Maruyama, and A. Ohnishi, Phys. Rev. Lett. 68, (1992) 2898 .

[9] T. Maruyama, A. Ohnishi and H. Horiuchi, Phys. Rev. C42, (1990) 386.

[10] T. Maruyama, K. Niita, K. Oyamatsu, T. Maruyama, S. Chiba, and A. Iwamoto, Phys. Rev. C57, (1998) 655.

[11] A. Bonasera, J. J. Molitoris and F. Gulminelli, Phys. Rep. 243 (1994) 1.

[12] M. Papa et al., Phys. Rev. C 61 (2000) 044614. 